\title{
Ion-dipole interactions are asymptotically unscreened by water in dipolar nanopores, yielding patterned ion distributions
}

\author{
Kevin Leung \\ Sandia National Laboratories, MS 1415, \\ Albuquerque, NM 87185, kleung@sandia.gov
}

(Dated: November 8, 2018)

\begin{abstract}
The permeation, rejection, and transport of electrolytes in water-filled nanopores are critical to ion current gating and desalinalion processes in synthetic porous membranes and the functions of biological ion channels. While the effects of confinement pore polarizability, and discrete channel charge sites have been much studied, the potentially dramatic impact of dipole-lined synthetic pores on electrolytes has seldom been addressed. Dipole layers naturally occur on the interior surfaces of certain nanopores, leading to intrinsic preference for cations or anions. This preference can be exploited when the membrane surface is functionalized differently from the pore interior or when there are alternating dipolar/nondipolar stretches inside a long pore. The dipole-ion interaction is asymptotically unscreened by water, leading to ionic, charge segregated, insulating behavior that can block ion transport, and potentially novel current-voltage $(I-V)$ characteristics.
\end{abstract}


Water-filled nanopores exhibit a remarkable ability to exclude or selectively transmit ions. ${ }^{1-8}$ They have important biological functions, and are potentially useful for desalination and other applications. The effects of dielectric mismatch, ${ }^{1}$ nanopore diameter, ${ }^{2}$ net charge ${ }^{7}$ and polarizability ${ }^{4,5,9}$ on ion permeation and rejection have been much studied. Surprisingly, while the celebrated KcsA channel selectivity filter relies on carbonyl group dipoles pointed radially into its narrow passageway for its function, ${ }^{8}$ dipolar decorations in synthetic nanopores have received less attention. A recent force field-based model has however shown that synthetic silica nanopores contain dipoles on its interior surface and exhibit an intrinsic preference for cations - even at the $\mathrm{pH}$ of zero charge. ${ }^{6}$ This work elucidates the dramatic effect of dipolar layers in nanopores on electrolyte permeation, which may lead to novel current-voltage $(I-V)$ characteristics, and discusses pore geometries where this effect may be realized.

An infinitely long cylindrical pore of radius $R_{d}$ with radially aligned surface dipoles of magnitude $d$ and uniform surface density $\eta_{d}$ exerts a uniform electrostatic potential $\phi_{\mathrm{d}}=$ $-4 \pi \eta_{d} d$ in the pore interior, independent of $R_{d}$ or radial position of ion $R$. This intrinsic $\phi_{\mathrm{d}}|e|$ is predicted to be $\sim-24 \mathrm{kcal} / \mathrm{mol}$ in silica pores ${ }^{6}{ }^{6}$ and exceeds $-100 \mathrm{kcal} / \mathrm{mol}$ inside the KcsA filter. ${ }^{8,10}$ Here $e$ is the electronic charge. A uniform dipole layer thus induces no electric field and does not interact with uncharged $\mathrm{H}_{2} \mathrm{O}$ molecules. Hence its interaction with ions inside the pore is unmediated (unscreened) by the confined water. This is consistent with our hitherto puzzling observation that the free energy preference for $\mathrm{Na}^{+}$over $\mathrm{Cl}^{-}$in the infinitely long silica pore of Ref. 6 is fairly independent of the presence of water. Thus the simple uniform dipole density in our model apparently captures essential nanopore-ion interactions in nanoporous silica, where $\mathrm{H}_{2} \mathrm{O}$ molecules do form hydrogen bonds with surface silanol groups. This model should be pertinent to other inorganic nanopores too.

We next use two models to demonstrate how this intrinsic $\phi_{d}$ may be exploited: an infinitely long "tube" geometry, with repeating unit cells that have alternating $L / 2$ long stretches where dipoles surface densities exist or vanish (Fig. 1a); (2) a "membrane" geometry with dipoles in the pore interior only (Fig. 1b). Regions with low local dipole density can potentially be achieved by functionalizing silica pores with amine groups. ${ }^{6}$ Near the junction, $z=z_{o}$, between dipolar $\left(\phi=-4 \pi \eta_{d} d\right)$ and non dipolar $(\phi=0)$ regions, the pore-axis electrostatic potential is

$$
\phi_{\mathrm{d}}(z, R=0)=-2 \pi \eta_{d} d\left\{\frac{\left(z-z_{o}\right) / R_{\mathrm{d}}}{{ }^{\prime}}+1\right\}
$$


$\phi(z, R)$ is fairly independent of $R$ inside the pore but vanishes rapidly for $R>R_{\mathrm{d}}$. An electric field parallel to the pore interior surface materializes,${ }^{11}$ localized within a distance $R_{\mathrm{d}}$ of the entrance. This field is locally screened by water molecules.

Note that in the membrane model, if the membrane surface has the same dipole density as the pore interior surface, it exerts an equal and opposite $\phi_{\mathrm{d}}(z, R=0)$ that cancels Eq. $1 .^{10}$ Hence, to exploit dipolar effects, the two surfaces must be functionalized differently.

To examine ion permeation into water-filled dipolar pores, we apply the atomistic $\mathrm{H}_{2} \mathrm{O}$, $\mathrm{Na}^{+}$, and $\mathrm{Cl}^{-}$force fields used in Ref. 6 to compute the potential of mean force $W(z)$. Porewater/ion interactions are described by analytical, least square fit approximations to dipolar electrostatic and Lennard-Jones terms. ${ }^{10}$ The pores are hydrophilic, with $\sim 12 \AA$ diameter and $2.7 \AA^{-1} \mathrm{H}_{2} \mathrm{O}$ density. Infinitely long but laterally isolated tubes (Fig. 1a) are simulated using at least $10^{5}$ passes of canonical ensemble Monte Carlo (MC) runs. Membrane models (Fig. 1b), periodic in all 3 directions with lateral unit cell lengths of $40 \AA$, are simulated for at least 200 ps using molecular dynamics (MD)/particle mesh Ewald methods. Simulation cells contain two unit cells doubled in the $z$ direction. Because $\mathrm{Na}^{+}$orders water in nanpores in a way incompatible with the periodic boundary conditions used, ${ }^{12}$ our simulation cells contain two unit cells doubled in the $z$ direction for convergence.

Figure $2 \mathrm{~b}$ depicts $W(z)$ for a $\phi_{\mathrm{d}}=-72 \mathrm{kcal} / \mathrm{mol}$ and $L / 2=30 \AA$ tube. The membrane geometry leads to qualitatively similar results (Fig. 2c). These geometries yield $\Delta W_{1}=$ $W(L / 2)-W(0)=-8.1$ and $-12.5 \mathrm{kcal} / \mathrm{mol}$. Compared with $\Delta W_{1}=\phi_{d}=-72 \mathrm{kcal} / \mathrm{mol}$ in the absence of water, they exhibit effective dielectric constants of 8.9 and 6.0, respectively.

However, unlike transmembrane ion channels such as $\mathrm{KcsA},{ }^{8}$ material pores can have lengths on the order of $1 \mu \mathrm{m}$. Table I shows that $\Delta W_{1}$ in the tube geometry steadily increases with $L / 2$ and is roughly proportional to $\phi_{\mathrm{d}}$; at $L / 2=120 \AA, \epsilon \sim 2.3$. This is readily explained as follows. Unless the localized electric field induces a ferroelectric phase transition (not the case here), $\Delta W_{1}$ is predominantly screened by $\mathrm{H}_{2} \mathrm{O}$ near the junctions where the electric field at its maximum. For large $L$, these localized $\mathrm{H}_{2} \mathrm{O}$ resemble a point dipole whose interaction with ions vanishes as $1 /\left|z-z_{o}\right|^{2}$. Thus, the attraction of cations deep inside dipolar regions is asymptotically unscreened by water. $\Delta W_{1}$ analysis for the membrane geometry is complicated by its non-zero value even when the dipoles are absent in membrane slabs, and by the $O(1) \mathrm{kcal} / \mathrm{mol}$ statistical noise, but $\Delta W_{1}$ follows a qualitatively similar trend there. 
screen the ion-surface dipole interaction. Here nanoscale pores with $R_{d}<10 \AA$ may exhibit unique properties. (1) We consider $1.0 \mathrm{M} \mathrm{NaCl}$ electrolytes in the membrane geometry. The hydration free energy of the $\mathrm{Na}^{+}$in the middle of the dipolar pore is reduced (last lines of Table I) because other $\mathrm{Na}^{+}$ions now enter the nanopore, while $\mathrm{Cl}^{-}$seldom do; the resulting electrostatic potential increase makes cations less favorable. ${ }^{10}$ But unlike macroscopic pores, even the $1.0 \mathrm{M} \mathrm{NaCl}$ electrolytes do not yield complete screening in this membrane geometry. This is possibly due to confinement effects. Results from 2.7 and 5.1 ns MD trajectories with different initial configurations are used here because ions diffuse rather slowly. (2) Azobenzene ${ }^{13}$ or similar functional groups can be placed at the junctions where the axial electric field is largest, further restricting water density there and hindering screening. (3) Nanopores with alternating dipolar and non-dipolar surfaces may result in an ionic, charge segregated, insulating behavior.

We use MC to demonstrate that such a "phase separation" occurs with a confined $0.5 \mathrm{M}$ $\mathrm{NaCl}$ inside an infinitely long pore with unit cell $L=240 \AA$ and $\phi_{\mathrm{d}}|e|=-72 \mathrm{kcal} / \mathrm{mol}$ (see Fig. 1c). The effective dipole-ion screening for this $L$ is 2.3 (Table I). This $\mathrm{Na}^{+}$concentration is qualitatively consistent with our simulation of permeation of $1.0 \mathrm{M} \mathrm{NaCl}$ into the nanopore in the membrane geometry. ${ }^{10}$ To accelerate ion sampling, water is here approximated as a dielectric with $\epsilon_{\mathrm{w}}=10$, likely slightly underestimated because this is obtained by considering a contact ion pair in the tube. ${ }^{10}$ We find that ion transport is blocked even upon applying a $0.0025 \mathrm{~V} / \AA$ electric field screened by $\epsilon_{\mathrm{w}}=10$. We stress that this insulating behavior arises from (1) the almost-unscreened ion-pore dipole interaction dominating the better-screened $\left(\epsilon_{w}=10\right)$, ion-ion coulomb interactions, and (2) the alternating dipolar regions leading to a complete absence of cation/anion along lengths of the nanopore. Future simulations of $I-V$ characteristics, taking into account leakage of $\mathrm{Na}^{+}$through the nondipolar regions at long times, will be profitable. ${ }^{14}$

In conclusion, dipole layers naturally occur in certain nanopores, leading to intrinsic preference for cations or anions. This preference can be exploited when the membrane surface is functionalized differently from the pore interior, or when there are alternating dipolar/nondipolar stretches inside a long pore. The dipole-ion interaction is asymptotically unscreened by water, potentially leading to novel ionic, charge segregated, insulating behavior that can block ion transport. Our finding may lead to new synthetic ion-exclusion membranes.

Sandia is a multiprogram laboratory operated by Sandia Corporation, a Lockheed Martin 
under contract DE-AC04-94AL8500.

1 Jordan, P.C.; Biophys. J. 1982, 39, 157; Parsegian, A. Nature 1969, 221, 844.

2 Beckstein, O.; Tai, K.-H.; Sansom, M.S.P. J. Am. Chem. Soc. 2004, 126, 14694; and references therein.

3 Kalra, A.; Garde, S.; Hummer, G. Proc. Natl. Acad. Sci. 2003, 100, 10175.

4 Peter, C.; Hummer, G. Biophy. J. 2006, 89, 2222.

5 Allen, R.; Melchionna, S.; Hansen, J.P. J. Phys. Condens. Matter 2003, 15, S297.

6 Leung, K; Rempe, S.B.; Lorenz, C.D. Phys. Rev. Lett. 2006, 96, 095504.

7 Stein, D.; Kruithof, M.; Dekker, C. Phys. Rev. Lett. 2004, 93, 035901; Gracheva, M.E.; Vidal, J.; Leburton, J.-P.; Nano Lett. 2007, 7, 1717; Lorenz, C.D.; Travesset, A., http://www.arXiv:0706.3514v1.

8 Varma, S.; Rempe, S.B. Biophys. J. 2007, 93, 1093; Noskov, S.R.; Roux, B. Gen. Physiol. 2007, 129, 135; Asthagiri, D.; Pratt, L.R.; Paulaitis, M.E. J. Chem. Phys. 2006, 125, 024701.

9 Leung, K; Marsman, M. J. Chem. Phys. 2007 (in press).

10 See Supporting Information available free of charge at http://pbs.acs.org.

11 Bratko, D.; Daub C.; Leung, K.; Luzar, A. J. Am. Chem. Soc. 2007, 129, 2504.

12 Dellago, C.; Naor, M.M.; Humer, G. Phys. Rev. Lett. 2003, , 105902.

13 Liu, N.G.; Dunphy, D.R.; Atanassov, P.; Bunge, S.D.; et al. Nano Lett. 2004, 4, 551.

14 Gillespi, D.; Nonner, W.; Eisenberg, R.S. J. Phys. Condens. Matter 2002, 14, 12129. 


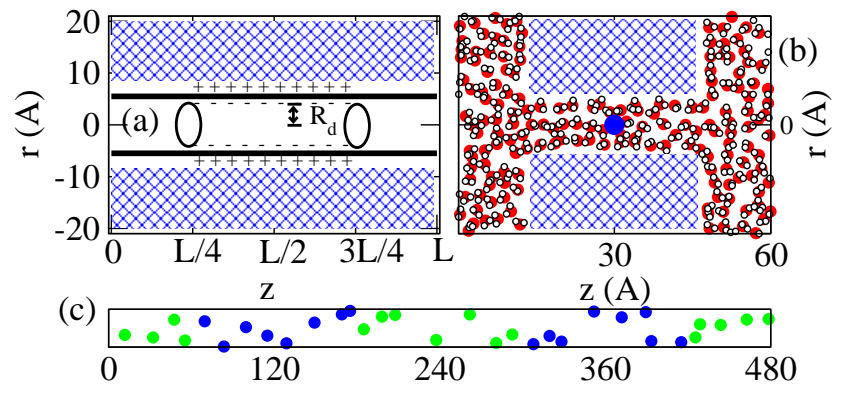

FIG. 1: (a) Cylindyrical nanopore unit cell for the "tube" geometry. Ions and water are excluded from the crossed regions. Radial dipole moments ("+/-") exist on alternating stretches along the pore surface. (b) "Membrane" simulation unit cell. Blue and red/white spheres depict $\mathrm{Na}^{+}$and $\mathrm{H}_{2} \mathrm{O}$ respectively. Dipoles exist on interior pore surface only. (c) Charge segregated insulating behavior in tube geometry (see text). $\mathrm{Na}^{+}$and $\mathrm{Cl}^{-}$are depicted as blue and green spheres. 


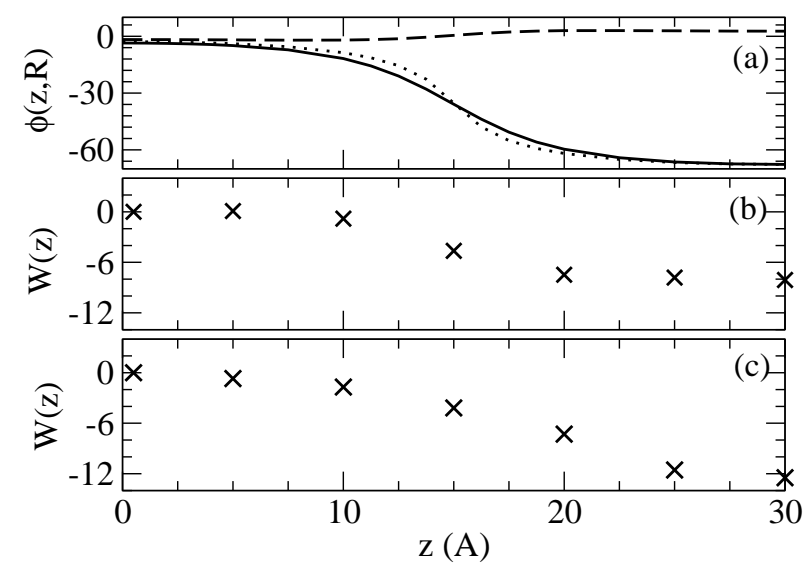

FIG. 2: $\mathrm{Na}^{+}$potential of mean force $W(z) \mathrm{kcal} / \mathrm{mol}$ along the pore axis, with $\phi_{\mathrm{d}}|e|=71.7 \mathrm{kcal} / \mathrm{mol}$, $L=60 \AA$, and a unit cell doubled in the $z$-direction. (a) No water; solid, dotted, and dashed lines refer to ions at radii $R / R_{\mathrm{d}}=0,0.7$, and 1.8 respectively. (b) Water-filled tube geometry. (c) Water-filled membrane geometry.

\begin{tabular}{|c|c|c|c|c|c|}
\hline \multicolumn{3}{|c|}{ tube } & \multicolumn{3}{|c|}{ mem. } \\
\hline$\phi_{\mathrm{d}}|e|$ & $L / 2$ & $\Delta W_{1}$ & $\phi_{\mathrm{d}}|e|$ & $L / 2$ & $\Delta W_{1}$ \\
\hline-71.7 & 30 & -8.1 & -71.7 & 30 & -12.5 \\
\hline-71.7 & 60 & -14.9 & -71.7 & 60 & -17.4 \\
\hline-71.7 & 90 & -24.0 & 0.0 & 30 & +3.3 \\
\hline-71.7 & 120 & -32.3 & 0.0 & 60 & +2.5 \\
\hline-23.9 & 60 & -3.0 & -23.9 & 30 & -1.7 \\
\hline \multirow[t]{3}{*}{-23.9} & 120 & -8.1 & 0.0 & 30 & +3.3 \\
\hline & & & -71.7 & 30 & -3.4 \\
\hline & & & 0.0 & 30 & +2.6 \\
\hline
\end{tabular}

TABLE I: $\mathrm{Na}^{+}$potential of mean force $\left(\Delta W_{1}\right)$ at $z=L / 2$, relative to its value at $z=0$ where $\phi_{\mathrm{d}} \approx 0$. Energies and lengths are in units of $\mathrm{kcal} / \mathrm{mol}$ and $\AA$. ${ }^{*}$ Computed in $1.0 \mathrm{M} \mathrm{NaCl}$ electrolyte, not pure water. 